\title{
Prevention of Mother to Child Transmission in HIV Antenatal and Postnatal Screening in Khulna Medical College
}

\author{
Shamsun Nahar*1, S. M. Khaliduzzaman², Parul Akter ${ }^{3}$, Zannatul Ferdous Jesmin ${ }^{4}$
}

\begin{abstract}
Introduction: Prevention of Mother to Child Transmission (PMTCT) program, offers a range of services for women of reproductive age living with or at risk of HIV to maintain health and stop their infants from acquiring HIV. PMTCT services should be commenced before conception and throughout pregnancy, labor and breast feeding. Around 1.4 million HIV infections among children were prevented between 2010 and 2018 by PMTCT program. UNFPO started antenatal screening program in collaboration with AIDS/STD program in Bangladesh from 2013, in 12 centers (BSMMU, 4 Medical College Hospitals, 3 Sadar Hospitals, 3 Health Complexes and Memon Maternity Hospital, Chittagong). Materials and Methods: Observational study of Antenatal and postnatal cases in HIV screening in KMCH during the period of Nov'2017 to March'2020. Results: From November 2017 to March 2020, total 18,911 patients screened, 11 patients were found HIV positive. 2 patients dropped out. Among 9 patients, 6 patients were detected in antenatal and 3 in postnatal period. 6 patients had vaginal delivery, 2 elective caesarean section and 1 patient had abortion. Screening was done by Alere Determine HIV-1/2 Ag/Ab Combotest and diagnosis was confirmed by Uni-Gold HIV Rapid Test and First Response HIV 1-2-0 Card test. Conclusion: Following confirmation, ARV started with proper counseling. All babies were breast fed. The neonates were screened by PCR at their age of 45 days and found negative. WHO guideline was strictly maintained during labor conduction and cesarean sections. PMTCT program should be expanded in all institutions to detect cases, reduce transmission, contraception counselling to reduce confirmed cases.
\end{abstract}

Keywords: PMTCT.

Number of Table: 01; Number of Figures: 03; Number of References: 16; Number of Correspondence: 04.

*1. Corresponding Author:

Prof. Shamsun Nahar, FCPS, MS

Professor and Head

Department of Obstetrics and Gynecology

Khulna Medical College (KMC), Khulna.

Email: gynaekmc@gmail.com

2. Dr. S. M. Khaliduzzaman, MS

Assistant Professor

Department of Obstetrics and Gynecology

Khulna Medical College (KMC), Khulna.

3. Dr. Parul Akter, FCPS

Indoor Medical Officer

Khulna Medical College and Hospital (KMCH), Khulna.

4. Dr. Zannatul Ferdous Jesmin, FCPS, DGO

Assistant Professor

Department of Obstetrics and Gynecology

Khulna Medical College (KMC), Khulna.

\section{Introduction:}

ON 5 JUNE 1981, A REPORT IN THE MORBIDITY AND MORTALITY WEEKLY REPORT (MMWR), no one who read those reports, certainly not this author, could have imagined that this was the first glimpse of a historic era in the annals of global health ${ }^{1}$. CDC defines a case of AIDS as a disease, at least moderately predictive of a defect in cell mediated immunity, occurring in a person with no known cause for diminished resistance to that disease ${ }^{2}$. Acquired immune deficiency syndrome, a disease in which there is a severe loss of the body's cellular immunity, greatly lowering the resistance to infection and malignancy ${ }^{3}$. The cause is a virus (the human immunodeficiency virus, or HIV) transmitted in blood and in sexual fluids. a disease of the human immune system that is characterized cytologically especially by reduction in the numbers of CD4-bearing helper T cells to 20 percent or less of normal thereby rendering the subject highly vulnerable to life-threatening conditions (as Pneumocystis carinii pneumonia) and to some (as Kaposi's sarcoma) that become life-threatening and that is caused by infection with HIV commonly transmitted in infected blood especially during illicit intravenous drug use and in bodily secretions (as semen) during sexual intercourse. The human immunodeficiency virus (HIV) eats away at the T-cells of the body's immune system, thereby exposing it to infections ${ }^{4}$. HIV is associated with increased rates of preterm delivery, low birth weight and stillbirth. Special intervention to reduce maternal to child transmission (MTCT) includes Anti-retro viral (ARV) treatment and prophylaxis, safer delivery procedures and counseling on safe breast feeding.

High viral load, low CD4 count, advanced HIV disease, presence of sexually transmitted diseases as well as prolonged labor and delivery specially when associated with chorioamnionitis increase transmission of the infection to the baby. The rate of transmission is $17.4 \%$, during pregnancy, 50\% during labor and delivery and the remaining $33 \%$ during breast feeding ${ }^{5}$. Elective CS is best done at 38 weeks especially when the viral load is greater than 1000 copies $/ \mathrm{ml}^{6}$. The WHO criteria for initiation of ARV therapy in adults are stage IV, stage IIl with CD4 $<350$ cells $/ \mathrm{mm}^{3}$, stage 1 and 2 when CD4 is < 200cells $/ \mathrm{mm}^{3,7} \mathrm{CD} 4$ count typically initially increases by greater than 50 cells $/ \mathrm{mm}^{3}$ at four to eight weeks in response to HAART and 
by an additional 50-to 100 per year thereafter. Family counseling is mandatory when patient is started on ARV treatment ${ }^{8}$. Th is study helps to detect HIV infected patients and initiation of treatment as early as possible with safe delivery and neonatal care, also to emphasize the importance of HIV screening in antenatal period for prevention of maternal to child transmission.

\section{Materials and Methods:}

Observational study of Antenatal and postnatal cases in HIV screening in KMCH during the period of Nov'2017 to March'2020. Screening was done by finger prick and taking $50 \mu$ liter of blood on "Alere Determine HIV- $1 / 2$ $\mathrm{Ag} / \mathrm{Ab}$ Combo test kit and after 20 minutes it shows HIV reactive or non-reactive ('Rapid test') and in positive cases diagnosis was confirmed by, Uni-Gold HIV Rapid Test and First Response HIV 1-2-0 Card test kits by same procedure and if all three tests show reactivity, the women is confirmed as HIV positive.

\section{HIV positive detected cases \\ Cases newly detected}

Case no: 1

Mrs. Nasima Khatun, age 18 years, primi, detected HIV positive on 14/01/2018 at her 16th week of pregnancy during antenatal checkup in outpatient department by routine screening test and Anti-retroviral drug was started accordingly. She was diagnosed as a case of intrauterine death at 28 weeks of pregnancy. Vaginal delivery was conducted under proper protocol at $\mathrm{KMCH}$. She has been continuing her regular follow up and doing well. Her husband was HIV negative.

\section{Case no: 2}

Mrs. Khadiza Khatun, age 25 years, G2P1, a case of full-term pregnancy, delivered a healthy baby per vaginally, identified HIV positive on postnatal screening. Her husband was HIV negative and 1st baby was HIV positive. The patient was absconded prior to initiation of treatment.

\section{Case no: 3}

Mrs. Razia Khatun age 25 years, primi with 6 months pregnancy, identified as HIV positive in antenatal screening on 15/04/2019, anti-retroviral was started accordingly, her EDD was on $29 / 05 / 2019$, but she did not report two months following treatment. Her husband was HIV negative.

\section{Case no: 4}

Mrs. Tania Khatun, age 21 years, Primi with 1st trimester pregnancy, HIV screening was positive on 28/07/2019 during antenatal checkup, She delivered a healthy baby per vaginally on 25/02/2019, Her husband was HIV positive, On 45th day, PCR test was done for the baby and was negative.

\section{Case no: 5}

Mrs. Lima Khanom, age 19 years, delivered a healthy baby per vaginally in $\mathrm{KMCH}$, detected HIV positive on 05/10/2019 during post-natal screening, anti-retroviral was started, Her husband was HIV positive, On 45th day of the baby, PCR test was done and result was negative. The patient is under regular follow-up and she is doing well

\section{Case no: 6}

Mrs. Sonia Akhter age 20 years, a nurse, G2P1, detected HIV positive on 30/11/2019 during antenatal screening, her EDD was 29/07/2020, Anti-retroviral was started accordingly, but after two months of treatment she discontinued medication and follow-up. Her husband was HIV positive a 1st baby was negative.

\section{Case no: 7}

Mrs. Farzana Khatun, age 20 years, was detected HIV positive on 09/12/2019 during antenatal screening, next day elective cesarean section was done in $\mathrm{KMCH}$. Anti-retroviral was started accordingly. She did not breast feed her baby. Her husband was HIV negative. On 45th day of the baby, PCR was done and result was negative. She is with regular follow-up and doing well.

\section{Case no: 8}

Mrs. Nasima Khanom age 32, detected HIV positive on postnatal screening on 14/03/2020. She delivered a still born baby in $\mathrm{KMCH}$. Anti-retroviral drug was started accordingly.

\section{Known cases}

\section{Case 1:}

Mrs. Parul Khatun age 19 years, HIV positive 2 years back. P2. Last vaginal delivery in $\mathrm{KMCH}$ on $17 / 03 / 2018$. Husband is HIV positive. PCR test was done in two of her babies, which were negative.

\section{Case 2:}

Mrs. Sharmin Akhter age 22 years, HIV positive 5 years back. She had a vaginal delivery on 14/11/2018 in KMCH. Her husband is also HIV positive. On 45th day of her baby PCR test was done and result was negative. Antibody test is due on May'2020.

\section{Case 3:}

Mrs. Munni Akhter age 25 years, P2-1 (NND) HIV positive 3 years back. Elective cesarean section on 14/04/2019 in $\mathrm{KMCH}$. Her 1st husband was HIV positive. Present husband is HIV negative. On 45th day of the baby, PCR was done and result was negative.

\section{Results:}

During the period of Nov'2017 to March'2020, we had screened for HIV in 18911 patients (ante natal and post-natal) in outpatient department and indoor obstetric ward. Screening was done by 'Rapid test' ("Alere Determine HIV- 1/2 Ag/Ab Combo). In positive cases, diagnosis was confirmed by, Uni-Gold HIV Rapid Test and First Response HIV 1-2-0 Card test. Total 11 patients were found HIV positive. Among them 3 ante natal patients were known cases with ongoing follow up for Anti-Retroviral (ARV) treatment. Two patients delivered per vaginally spontaneously and one patient underwent elective cesarean section. Remaining new 8 patients were detected HIV positive during ante natal period. Among them 5 patients 
delivered per vaginally spontaneously, one patient underwent elective cesarean section, one patient was absconded and one patient is still in ante natal period. Total 10 patients were offered Anti-Retroviral drugs (ARV) following confirmation of the diagnosis. 1st PCR test was done in 9 neonates on 45th day and Rapid test was done on 18th month of their age. They were found HIV -ve. PCR is due for them at their age of one year. Besides KMC, PMTCT activities are also ongoing in BSMMU, 4Medical Colleges, 3 Sadar Hospitals, 3 Upazilla Health Complexes and 1 private institution which is shown in Table No. I.

Table-I: PMTCT activities in different institutions throughout Bangladesh till June'2020.

\begin{tabular}{|c|c|c|c|c|c|c|c|}
\hline $\begin{array}{l}\text { SL } \\
\text { No. }\end{array}$ & $\begin{array}{l}\text { Name of } \\
\text { PMCT } \\
\text { center }\end{array}$ & 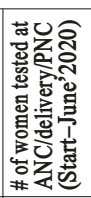 & 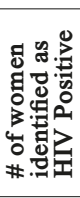 & 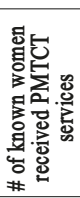 & 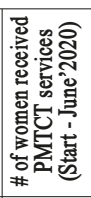 & 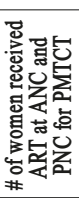 & 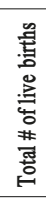 \\
\hline 1 & BSMMU \& SSMCH & 36207 & 13 & 85 & 98 & 94 & 78 \\
\hline 2 & SOMCH \& MDH & 77375 & 14 & 46 & 60 & 55 & 53 \\
\hline 3 & $\begin{array}{l}\text { CMCH, CGH \& } \\
\text { Memon Hospital }\end{array}$ & 89803 & 4 & 14 & 18 & 18 & 19 \\
\hline 4 & CDSH \& UUHC & 30493 & 24 & 16 & 40 & 40 & 29 \\
\hline 5 & $\mathrm{KMCH} \&$ Jashore & 23902 & 8 & 3 & 11 & 10 & 7 \\
\hline
\end{tabular}

Total screened patients were 257780 and detected HIV positive was $68(0.026 \%)$ which is shown in Fig No 1 .

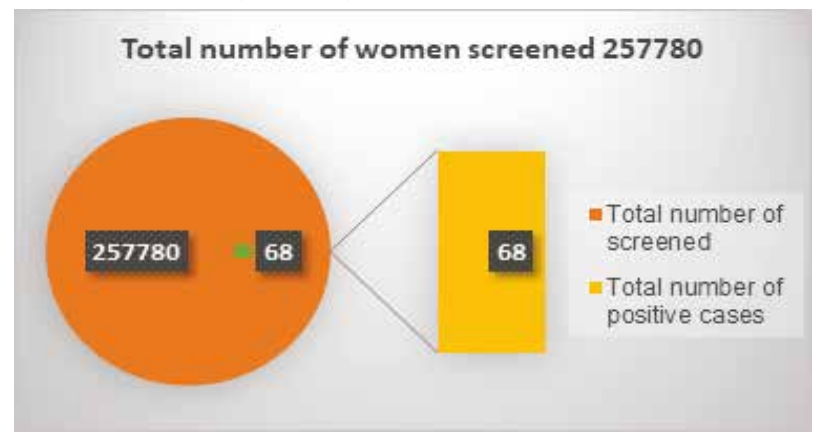

Fig - 1: Showing total number of screened patients and detected cases.

Total number of screened patients in $\mathrm{KMCH}$ were 189119 and detected HIV positive was $11(0.058 \%)$ which is shown in Fig No 2, Among 9 HIV positive cases, mode of pregnancy outcome was, NVD 6, elective LUCS 2 and abortion 1, which is shown in Fig No. 3.

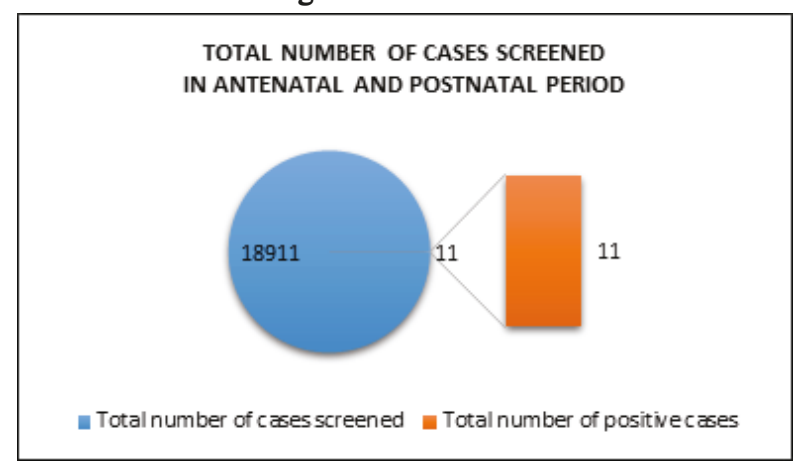

Fig -2: Showing total number of screened patients and detected cases in $\mathrm{KMCH}$.

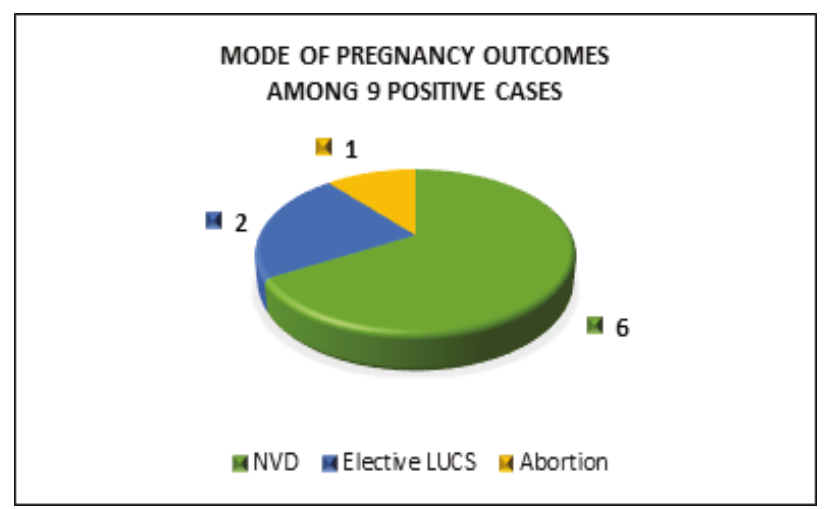

Fig -3: Showing mode of pregnancy outcomes among 9 positive cases.

\section{Discussion:}

HIV can be transmitted from an HIV-positive woman to her child during pregnancy, child birth and breast feeding. Mother to child transmission is also known as "vertical transmission', accounts for the vast majority of infections in children (0-14 years). Without treatment, if a pregnant woman is living with HIV the likelihood of the virus from mother-to-child transmission is $15-45 \%$. However, antiretroviral treatment (ART) and other interventions can reduce the risk below $5 \%{ }^{9}$.

World Health Organization (PMTCT) guideline includes: ${ }^{10}$

- preventing new HIV infections among women of reproductive age

- preventing unintended pregnancies among women living with HIV

- preventing HIV transmission from a woman living with HIV to her baby

- providing appropriate treatment, care and support to mothers living with HIV and their children and families.

Guideline for pregnant women living with HIV

In September 2015 WHO released guidelines recommending that all pregnant women living with HIV be immediately provided with lifelong treatment, regardless of CD4 count ${ }^{11}$.

\section{Guidelines on infant feeding for mothers living with HIV}

WHO bases its recommendations on infant feeding for mothers living with HIV on the comparative risk of infants acquiring HIV through breastfeeding with the increased risk of infants dying from illnesses such as malnutrition, diarrhea and pneumonia, which increases if they are not breastfed.

In 2016, WHO released guidelines recommending that mothers living with HIV who are on treatment and are being fully supported to adhere to it should exclusively breastfeed their infants for the first six months of life, then introduce appropriate complementary foods while continuing to breastfeed for at least 12 months and up to 24 months or longer (similar to the general population) ${ }^{12}$. 
Currently, most high-income countries recommend women living with HIV do not breastfeed whether they are virally suppressed or not. This is because formula feed and clean, boiled water are widely accessible. So, any risks around dirty water or malnutrition have been eliminated. In lowand middle-income countries this risk is far greater, leading WHO's advice on infant feeding to differ.

\section{Guidelines for HIV-exposed infants}

If an HIV-exposed infant is given ART within the first 12 weeks of life, they are $75 \%$ less likely to die from an AIDS-related illness ${ }^{13}$.

This is one of the reasons WHO recommends that infants born to mothers living with HIV are tested between four and six weeks old. This is often referred to as 'early infant diagnosis" ${ }^{\prime 4}$.

WHO further recommends that another HIV test is carried out at 18 months and/or when breastfeeding ends to provide the final infant diagnosis ${ }^{15}$. As proportionally more infant infections are now occurring during breastfeeding these tests are becoming increasingly important.

According to WHO guidelines, all infants who test positive for HIV should be immediately initiated on treatment. The treatment should be linked to the mother's course of ARV drugs and would vary according to the infant feeding method as follows:

- breastfeeding: the infant should receive once-daily nevirapine from birth for six weeks

- replacement feeding: the infant should receive once-daily nevirapine (or twice-daily zidovudine) from birth for four to six weeks ${ }^{15}$.

\section{Early infant diagnosis}

HIV positive infants and children who start treatment late are more likely to experience treatment failure, which underlines the need to diagnose HIV as early as possible ${ }^{16}$.

Providing treatment and care for HIV positive infants and children

There is an urgent need to accelerate treatment for children aliving with HIV across the priority countries. Globally in 2017, just over half (52\%) of 1.8 million children living with HIV were receivingtreatment.11This is far below the target of 1.6 million children on treatment by the end of 2018, as adopted in the 2016 Political Declaration on Ending AIDS ${ }^{16}$.

UNFPA in collaboration with AIDS/STD program started screening test in Bangladesh for 2013 and till now, it's activity is extended in different govt. and non govt institutions including BSMMU, 4 Medical College Hospitals, 3 Sadar Hospitals, 3 Health Complexes and Memon Maternity Hospital, Chittagong. Total 257780 patients were screened and total 64 patients ware confirmed as HIV positive (both in antenatal and postnatal period). In $\mathrm{KMCH}$, total 18911 patients were screened and total 11 cases were confirmed HIV positive, WHO protocol was maintained regarding NVD and elective cesarean section. All patient received ARV as early as the diagnosis was confirmed during antenatal and postnatal period. All mothers offered breast feeding to their babies. All HIV positive patient are from low socioeconomic status and disease was transmitted from their husbands (heterosexual transmission) and by blood transfusion. ARV also offered to all babies and PCR was done on their age of 45th day and all results were negative. So, the vertical transmission was zero.

Pregnancy perse has got no effect on the disease progression in HIV positive women. Increased incidence of abortion, prematurity, preeclampsia, IUGR and perinatal mortality in HIV seropositive mothers still remains inconclusive. Among the HIV positive cases in $\mathrm{KMCH}$, only two abortions occurred, so the overall pregnancy outcome was remarkable.

The initial presentation of an infected patient may be fever, malaise, headache, sore throat, lymphadenopathy and maculopapular rash. Majority of positive cases were asymptomatic, only one case presented with low grade fever, diarrhea and severe weight loss.

All patients are under regular follow-up and ARV was provided, the disease progression and effectivity of ARV are measured by PCR (detects viral load) and CD4 + cell count (detects maternal immune status). High cost of mentioned investigations hinders the patient's acceptance of follow-up procedure.

\section{Conclusion:}

Expansion of the PMTCT program is now mandatory, all institutions from primary to tertiary level, both government and non-government should start PMTCT activities, at least screening of all pregnant women (antenatal and post-natal period), starting ARV on the instant of confirmation of diagnosis, following proper protocol during labor management, proper postnatal follow up and ARV treatment outcome. Thus, there will be reduction of transmission of disease, reduction of mortality and morbidity of confirmed cases, reduction of transmission to babies will offer the healthy generations as well as healthy planet.

\section{Conflict of Interest: None.}

\section{Acknowledgement:}

We are grateful and expressing in-depth thanks to those who are doing their best job in this PMTCT program. Also privileged getting valuable comment and suggestions form the editor.

\section{References:}

1. Fanci AS. Twenty-five years of HIV-AIDS. Science Published by AAAS. 2006; 315:409.

https://doi.org/10.1126/science.1131993

PMid:16873613

2. Holmes CB, Losina E, Walensky RP, Yazdanpanah Y, Freedberg KA . Review of human immunodeficiency virus type 1-related opportunistic infections in sub-Saharan 
Africa. Clin. Infect. Dis. 2003; 36 (5): 656-662.

https://doi.org/10.1086/367655

PMid: 12594648

3. Ho-Yen C and Chang F. Gastrointestinal Malignancies in HIV/AIDS. The AIDS Reader. 2008; 18 (6): 300-302.

4. Charles Gilks. What Use is a Clinical Case Definition for AIDS in Africa? Br Med J. 1991;303: 1190.

https://doi.org/10.1136/bmj.303.6811.1189

PMid:1747621 PMCid:PMC1671473

5. Barlett John G. M, D \& Gallant Joel E., M.D, M.P.H. Medical management of HIV infection, South African Edition, Durham, NC, United States of America, TheraSim Inc; 2008.

6. Marcollet Anne MD, GoffinetFrançois MD, FirtionGhislaine MD. 'Differences in postpartum morbidity in women ........................ emergency cesarean delivery, or vaginal delivery'. American Journal of Obstetrics and Gynecology. 2002 April; 186(4):784-789.

https://doi.org/10.1067/mob.2002.122251

PMid: 11967508

7. Jennifer S. Read, Ruth Tuomala, Eloi Kpamegan. Mode of Delivery and Postpartum Morbidity among HIV-Infected Women: The Women and Infants Transmission Study. JAIDS Journal of Acquired Immune Deficiency Syndromes. 2001; 26:236-245.

https://doi.org/10.1097/00126334-200103010-00005

https://doi.org/10.1097/00042560-200103010-00005

PMid:11242196

8. Patrick G. Yeni, MD; Scott M. Hammer, MD; Charles C. J. Carpenter, MD. Antiretroviral Treatment for Adult HIV Infection in 2002. Updated Recommendations of the International AIDS Society-USA Panel JAMA. 2002; 288(2):222-235. https://doi.org/10.1001/jama.288.2.222

PMid:12095387

9. World Health Organization (WHO) HIV/AIDS Topical information Mother-to-child transmission of HIV [cited November 2018] Available from https://www.who.int/hiv/topics/mtct/about/en/

10. World Health Organization (2010) 'PMTCT strategic vision 2010-2015: Preventing mother-to child transmission of HIV to reach the UNGASS and Millennium Development Goals 'WHO Press, World Health Organization, 20 Avenue Appia, 1211 Geneva 27, Switzerland: page 9

11. World Health Organization (WHO) (2015) 'Guideline on when to start antiretroviral therapy and on pre-exposure prophylaxis for HIV' September 2015

12. World Health Organization 2016 GUIDELINE UPDATES ON HIV AND INFANT FEEDING, WHO Press, 20 Avenue Appia, 1211 Geneva 27, Switzerland. available on the WHO website (http://www.who.int) 13. UNAIDS/JC2656 'The Gap Report' (English original, July 2014, updated September 2014) ISBN 978-92-9253-062-4

14. UNAIDS Joint United Nations Programme on HIV/AIDS "CHILDREN AND HIV Fact sheet" 20 Avenue Appia 1211 Geneva 27 Switzerland

15. World Health Organization 2015 "Guideline on when to start antiretroviral therapy and on pre-exposure prophylaxis for HIV" WHO Press, World Health Organization, 20 Avenue Appia, 1211 Geneva 27, Switzerland

16. Joint United Nations Programme on HIV/AIDS (UNAIDS) 2017 "Start Free Stay Free AIDS Free 2017 progress report" 20 Avenue Appia 1211 Geneva 27 Switzerland 